\title{
A role for intracellular zinc in glioma alteration of neuronal chloride equilibrium
}

\author{
S Di Angelantonio, ${ }^{*, 1,2}$, E Murana ${ }^{1}$, S Cocco ${ }^{1}$, F Scala ${ }^{1}$, C Bertollini ${ }^{1}$, MG Molinari ${ }^{1}$, C Lauro ${ }^{1}$, P Bregestovski ${ }^{3}$, C Limatola ${ }^{1,4}$ \\ and D Ragozzino ${ }^{1,4}$
}

Glioma patients commonly suffer from epileptic seizures. However, the mechanisms of glioma-associated epilepsy are far to be completely understood. Using glioma-neurons co-cultures, we found that tumor cells are able to deeply influence neuronal chloride homeostasis, by depolarizing the reversal potential of $\gamma$-aminobutyric acid (GABA)-evoked currents $\left(\mathrm{E}_{\mathrm{GABA}}\right)$. $\mathrm{E}_{\mathrm{GABA}}$ depolarizing shift is due to zinc-dependent reduction of neuronal KCC2 activity and requires glutamate release from glioma cells. Consistently, intracellular zinc loading rapidly depolarizes $\mathrm{E}_{\mathrm{GABA}}$ in mouse hippocampal neurons, through the Src/Trk pathway and this effect is promptly reverted upon zinc chelation. This study provides a possible molecular mechanism linking glioma invasion to excitation/inhibition imbalance and epileptic seizures, through the zinc-mediated disruption of neuronal chloride homeostasis. Cell Death and Disease (2014) 5, e1501; doi:10.1038/cddis.2014.437; published online 30 October 2014

Glioma-associated epilepsy is an established but poorly understood phenomenon. Over $80 \%$ of glioma patients suffer from seizures, ${ }^{1}$ often representing the first symptomatic presentation of a tumor and possibly preceding it. ${ }^{2}$ It has been extensively reported that glioma cells release glutamate in the extracellular space, through the glutamate-cystine transporter (system Xc), promoting proliferation and invasion and causing neuronal death. ${ }^{3}$ Accordingly, increased glutamate levels have been implicated in numerous seizure disorders ${ }^{4}$ and contribute to epileptogenesis in gliomaimplanted rodents. ${ }^{5,6}$ Glutamate excess may cause the alteration of neuronal chloride $\left(\mathrm{Cl}^{-}\right)$homeostasis and depolarize $\gamma$-aminobutyric acid (GABA) reversal potential $\left(\mathrm{E}_{\mathrm{GABA}}\right)$, as a result of $\mathrm{Cl}^{-}$transporters dysfunction or disequilibrium. ${ }^{7}$ Indeed, a precise balance between NKCC1 and KCC2 activity is necessary for inhibitory GABAergic signaling in the adult central nervous system ${ }^{8}$ and its disequilibrium can cause elevation of intracellular $\left[\mathrm{Cl}^{-}\right]$leading to switch of GABAergic signaling from hyperpolarizing to depolarizing in epileptic tissue ${ }^{9,10}$ contributing to epileptogenesis. ${ }^{11-13}$

In this study, we investigated the mechanisms of gliomainduced neuronal overexcitation using co-cultures of hippocampal and glioma cells. We report that glioma cells cause the alteration of $\mathrm{E}_{\mathrm{GABA}}$, through glutamate-receptor-dependent zinc $\left(\mathrm{Zn}^{2+}\right)$ accumulation, leading to $\mathrm{KCC} 2$-mediated $\mathrm{Cl}^{-}$ transport unbalance. Our study provides the molecular mechanism of glioma-induced elevation in intracellular $\mathrm{Cl}^{-}$ and a complete model linking glutamate release by glioma cells to glioma-related epilepsy.

\section{Results}

Glioma co-culture increases neuronal $\left[\mathrm{Cl}^{-}\right]_{\mathrm{i}}$ by a glutamatergic mechanism. To address the effect of glioma cells on neuronal $\mathrm{Cl}^{-}$equilibrium, we determined the reversal potential of the currents evoked by GABA application in mouse hippocampal cultured neurons. As shown in Figures $1 \mathrm{a}$ and $\mathrm{b}$, co-culturing neurons with patient-derived glioma cells (MZC) ${ }^{14}$ caused a rightward shift in the current-voltage relationship of GABA-mediated responses, giving a positive shift of $E_{\text {GABA }}$ from $-73.9 \pm 1.2 \mathrm{mV}$ (control; $n=124$ ) to $-52.1 \pm 1.6 \mathrm{mV}$ (co-culture; $n=101, P<0.001$ ).

In control neurons, $\mathrm{E}_{\mathrm{GABA}}$ was significantly below resting membrane potential (RMP); conversely, in co-cultured neurons, despite a small depolarization of resting potential (Figure $1 \mathrm{~b}$ ), $\mathrm{E}_{\mathrm{GABA}}$ value was consistently more positive than RMP, reverting the driving force for GABA-mediated currents (Figure 1c). Similar results were observed also after a shorter (4h) co-culture duration $\left(\mathrm{E}_{\mathrm{GABA}}=-52.2 \pm 4.4 \mathrm{mV} ; n=18\right)$. $E_{G A B A}$ shift resulted from the increase in $\left[\mathrm{Cl}^{-}\right]_{\text {i }}$, as directly demonstrated using a genetically encoded Cl-Sensor, ${ }^{15,16}$ which gave values similar to those calculated by Nernst equation (Figure 1d).

To investigate the possible role of glutamate released by glioma cells in $\mathrm{E}_{\mathrm{GABA}}$ depolarization, glutamate receptors (GluRs) antagonists (D-(-)-2-Amino-5-phosphonopentanoic acid (APV), $20 \mu \mathrm{M}$; 2,3-Dioxo-6-nitro-1,2,3,4-tetrahydrobenzo [f]quinoxaline-7-sulfonamide disodium salt (NBQX), $10 \mu \mathrm{M}$ ) were added to the co-culture medium. In this condition,

\footnotetext{
${ }^{1}$ Istituto Pasteur-Fondazione Cenci Bolognetti, Department of Physiology and Pharmacology, Sapienza University of Rome, Piazzale Aldo Piazzale Aldo Moro 5, Roma 00185, Italy; ${ }^{2}$ Center for Life Nano Science@ Sapienza, Istituto Italiano di Tecnologia, Viale Regina Elena 291, Roma 00161, Italy; ${ }^{3}$ INSERM URM 1106, Aix-Marseille University, Brain Dynamics Institute, Marseille, France and ${ }^{4}$ IRCCS Neuromed, Via Atinese, Pozzilli, Italy

*Corresponding author: S Di Angelantonio, Department of Physiology and Pharmacology, Sapienza University of Rome, Piazzale Aldo Moro 5, Roma 00185, Italy. Tel: +390649910971; Fax: 390649910951; E-mail: silvia.diangelantonio @ uniroma1.it

Abbreviations: $\mathrm{E}_{\mathrm{GABA}}$, Reversal potential of GABA-evoked currents; system Xc, glutamate-cystine transporter; APV, D-2-amino-5-phosphonopentanoic; [CI-]l, chloride concentration; DMSO, dimethyl sulfoxide; GABA, $\gamma$-aminobutyric acid; GCM, glioma-conditioned medium; Glu, glutamate; NBQX, 2,3-dihydroxy-6-nitro-7-sulfamoyl-benzo [f]quinoxaline-2,3-dione; RMP, resting membrane potential; DIOA, R-(+)-butylindazone dihydroindenyl-oxy alkanoic acid; TPEN, N,N,N', $\mathrm{N}^{\prime}$-tetrakis(2-pyridylmethyl) ethylenediamine

Received 07.4.14; revised 04.9.14; accepted 09.9.14; Edited by A Verkhratsky
} 


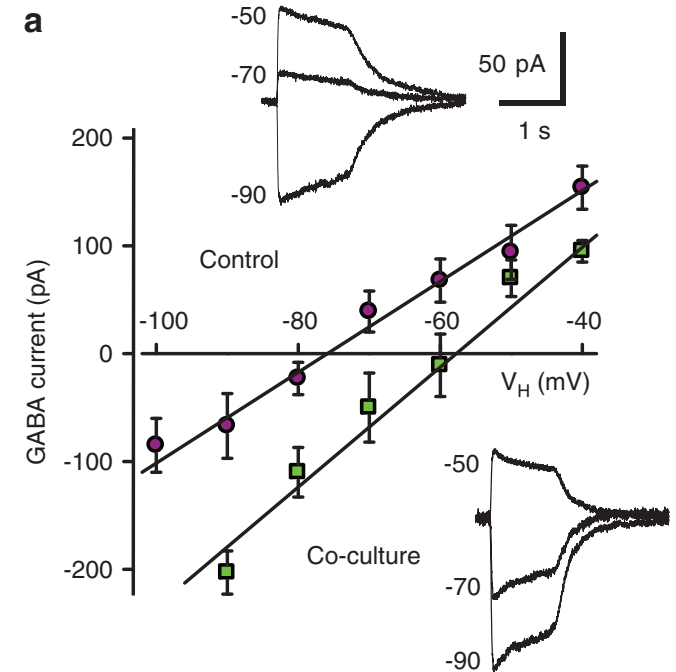

C

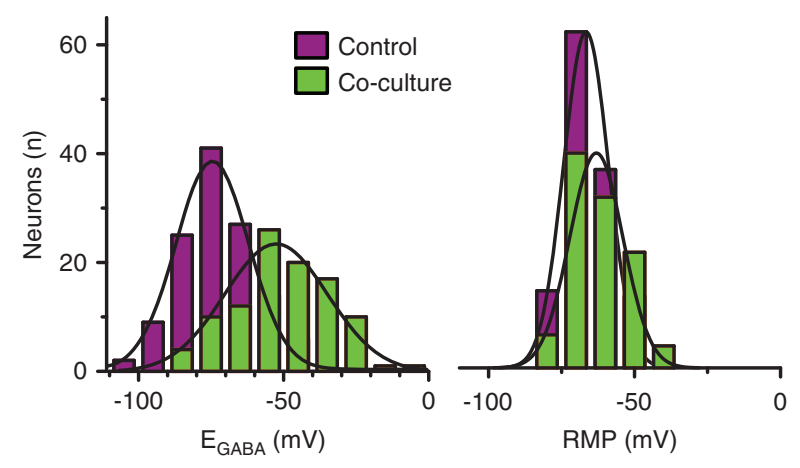

b
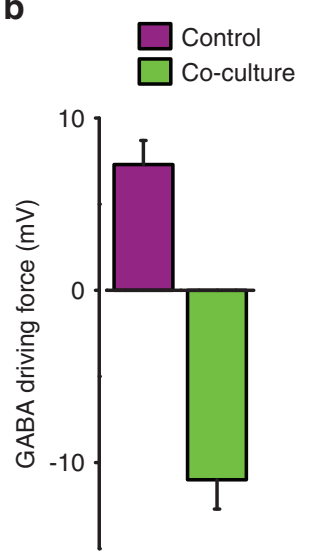

d

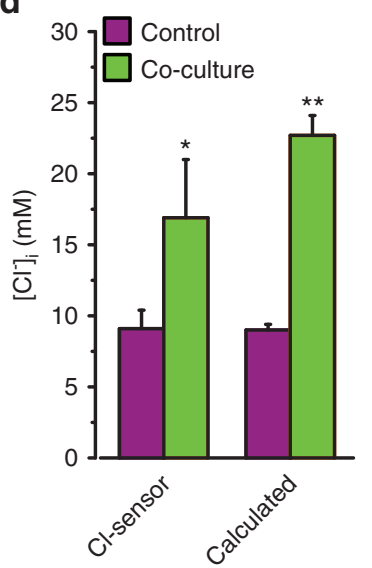

Figure 1 Glioma co-culture increases basal $\left[\mathrm{Cl}^{-}\right]$i in mouse hippocampal neurons depolarizing $\mathrm{E}_{\mathrm{GABA}}$. (a) Average I-V relation of GABA-activated currents recorded by perforated patch in control ( $n=24$; purple circles) and glioma co-cultured (24h,1:1 ratio; $n=18$; green circles) neurons (12 DIV); typical traces recorded in control and cocultured neurons at indicated membrane potentials; (b) Left, distributions of $\mathrm{E}_{\mathrm{GABA}}$ in control (purple columns) and co-cultured neurons (green columns; average $\mathrm{E}_{\mathrm{GABA}}$, control $-73.9 \pm 1.2 \mathrm{mV}$, co-culture $-52.1 \pm 1.6 \mathrm{mV} ; n=124 / 101, P<0.001 \mathrm{ANOVA}$ ); Right, distributions of RMP in control (purple columns) and co-cultured neurons (green columns; average RMP, control $-66.6 \pm 0.7 \mathrm{mV}$, co-culture $-63.1 \pm 0.9 \mathrm{mV} ; n=124 / 101, P<0.05$ ANOVA); (c) Driving force for GABA (RMP-E $E_{\text {GABA }}$ ) (control/co-culture as in (b); $n=124 / 101 ; P<0.001$ unpaired $t$-test); (d) basal neuronal $\left[\mathrm{Cl}^{-}\right]_{i}$ measured in neurons transfected with $\mathrm{Cl}$-Sensor (control, $\left[\mathrm{Cl}^{-}\right]_{i}=9.1 \pm 1.2 \mathrm{mM}$, purple column $n=243$; co-cultures, $\left[\mathrm{Cl}^{-}\right]_{\mathrm{i}}=16.9 \pm 4.1 \mathrm{mM}$; green column, $n=43$ ), and calculated using Nernst equation (control, $n=124 ;$ co-culture, $n=101 ; P<0.001$ unpaired $t$-test)

glioma-induced $\mathrm{E}_{\mathrm{GABA}}$ depolarizing shift was prevented (Figure 2a). Similar effects were observed when co-culture experiments were performed in the sole presence of APV $\left(\mathrm{E}_{\mathrm{GABA}}=-70.6 \pm 1.96 \mathrm{mV} ; \quad n=12, \quad P<0.01\right)$ or $\mathrm{NBQX}$ $\left(\mathrm{E}_{\mathrm{GABA}}=-70.5 \pm 7.7 ; n=6, P<0.01\right)$, indicating that activation of both AMPA and NMDA receptors is necessary to cause $\mathrm{E}_{\mathrm{GABA}}$ shift.

In addition, when the system $X_{c}$ blocker sulfasalazine $(250 \mu \mathrm{M})$ was added to the co-culture medium, $\mathrm{E}_{\mathrm{GABA}}$ depolarization was prevented (Figure 2a), showing that this effect requires $\mathrm{Xc}$-mediated glutamate release.

Moreover, acutely applied glioma-conditioned medium (GCM) activated APV/NBQX-sensitive inward currents (Figure 2b) in hippocampal neurons, confirming the presence of GluRs agonists in GCM. ${ }^{17}$

Altogether, these data show that in the co-culture conditions, glioma cells cause the depolarizing shift of $\mathrm{Cl}^{-}$ equilibrium potential in neurons, through glutamate release and GluRs activation. Consistently, $\mathrm{E}_{\mathrm{GABA}}$ depolarization was observed in neurons co-cultured with different human and murine glioma cells, but not with astrocytes (Figure 2c, and Supplementary Table S1), indicating a tumor-specific effect.

Glioma-induced $E_{\mathrm{GABA}}$ depolarization is due to $\mathrm{Cl}^{-}$ transporters unbalance. In cultured hippocampal neurons, KCC2 and NKCC1 transporters participate in the regulation of $\mathrm{Cl}^{-}$homeostasis. Indeed, the acute application of furosemide (100 $\mu \mathrm{M} ; 5-15 \mathrm{~min})$, the blocker of both transporters, caused a minor shift in neuronal $\mathrm{E}_{\mathrm{GABA}}$. Conversely, the specific blockers of NKCC1 (bumetanide; $10 \mu \mathrm{M}$; acutely applied 5-15 min) and KCC2 (R-(+)-butylindazone dihydroindenyl-oxy alkanoic acid, DIOA; $20 \mu \mathrm{M} ; 1 \mathrm{~h}$ pretreatment plus continuous superfusion) shifted $E_{G A B A}$ in opposite directions (Figure 3a), indicating that, in our culture conditions, both transporters actively regulate $\left[\mathrm{Cl}^{-}\right]_{\mathrm{i}}$.

The alteration of $\mathrm{Cl}^{-}$transporter activity is involved in glioma-induced $\mathrm{E}_{\mathrm{GABA}}$ shift in co-culture. In fact, in co-cultured neurons, furosemide, bumetanide or DIOA treatment abolished $E_{\text {GABA }}$ depolarization (Figure $3 b$ ). However, the acute application of bumetanide reverted the glioma-induced effect 
a
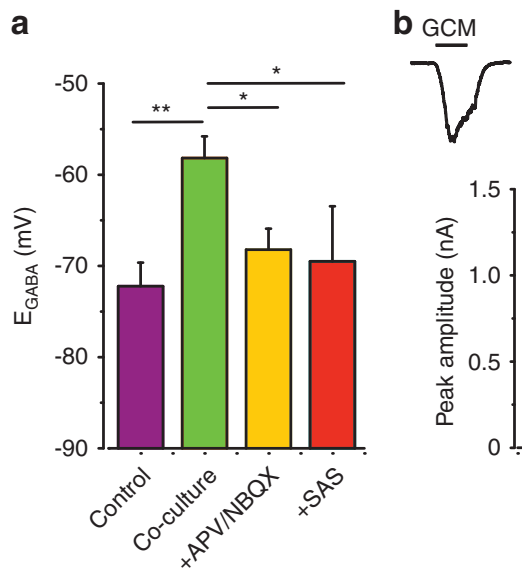

$\frac{\mathrm{GCM}}{\mathrm{APV/NBQX}}$
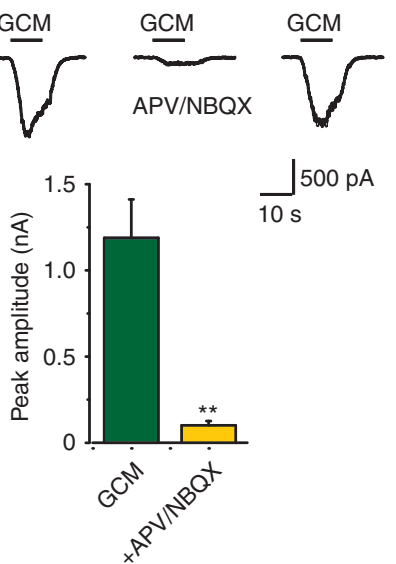

C

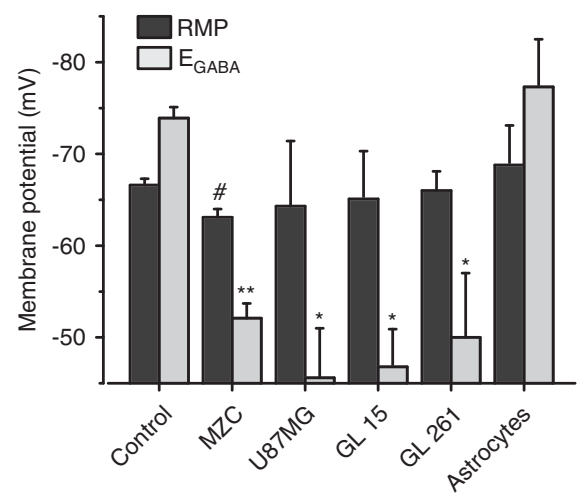

Figure $2 E_{G A B A}$ depolarization is due to glutamate release by glioma cells. (a) Inhibition of GluRs or transport prevents $E_{\text {GABA }}$ shift in co-cultured neurons; $E_{G A B A}$ in glioma co-culture either untreated $(-58.2 \pm 2.4 ; n=35$; green), in the presence of APV $(20 \mu \mathrm{M})$ and NBQX $(10 \mu \mathrm{M})(-68.2 \pm 2.3 ; n=27 ; P<0.05$, ANOVA; yellow), or of Xc glioma glutamate transport system blocker, sulfasalazine $(250 \mu \mathrm{M}$; $-69.5 \pm 6.0 ; n=10 ; \quad P<0.05$, ANOVA; orange) (control $E_{G A B A}-72.2 \pm 2.6$; $n=15$; purple). (b) GCM activates glutamatergic currents; Top, currents elicited in hippocampal neurons by GCM are blocked by ionotropic glutamatergic antagonists (NBQX $10 \mu \mathrm{M}$; APV $40 \mu \mathrm{M}$ ) and recover upon drugs washout; bottom, average amplitude of GCM-induced current responses before and after treatment with NBQX/ $\operatorname{APV}\left(n=14\right.$; yellow). (c) RMP (dark grey columns) and $\mathrm{E}_{\mathrm{GABA}}$ (light gray columns) values in neurons co-cultured with different glioma cell lines; MZC ( $n=101)$, U87MG $(n=7), \mathrm{GL} 15(n=4), \mathrm{GL} 261(n=10)$ and astrocytes $\left(n=7 ;{ }^{*} P<0.05,{ }^{* *} P<0.01\right.$ versus control neurons, ANOVA). Notice that MZC co-culture caused slight RMP depolarization (\# $P<0.05)$. Data and the corresponding estimated $\left[\mathrm{Cl}^{-}\right]_{\mathrm{i}}$, calculated using Nernst equation, are reported in Supplementary Table S1

(Figures $3 b$ and $c$ ), hyperpolarizing $\mathrm{E}_{\mathrm{GABA}}$. Conversely, DIOA treatment significantly depolarized $\mathrm{E}_{\mathrm{GABA}}$ by itself, likely occluding co-culture depolarizing effect (Figure 3b). These data indicate that glioma-induced $\mathrm{E}_{\mathrm{GABA}}$ depolarization is due to the unbalance of cation-chloride transporters activity, likely due to $\mathrm{KCC} 2$ reduced function.

To disclose the effects of glioma co-culture on neuronal KCC2 protein expression, we performed immunoblot experiments, revealing that the level of transporter expression was similar in control and co-cultured neurons (Figure 3d), thus indicating that glioma-induced alteration of neuronal $\mathrm{Cl}^{-}$ homeostasis relies on functional KCC2 modulation, rather than on changes in expression.
Glioma co-culture causes KCC2 impairment by increasing intracellular $\mathrm{Zn}^{2+}$ in neurons. To investigate a possible role of intracellular $\mathrm{Zn}^{2+}$ on glioma-induced $\mathrm{E}_{\mathrm{GABA}}$ shift, ${ }^{18}$ we used the membrane permeant $\mathrm{Zn}^{2+}$ chelator $\mathrm{N}, \mathrm{N}, \mathrm{N}^{\prime}, \mathrm{N}$ '-tetrakis(2-pyridylmethyl)ethylenediamine (TPEN). TPEN application $(20 \mu \mathrm{M} ; 5-20 \mathrm{~min})$ caused the rapid recovery of neuronal $E_{\text {GABA }}$ from glioma-induced depolarization (Figure 4a). This result suggests that glioma-induced $\mathrm{E}_{\mathrm{GABA}}$ shift can be ascribed to KCC2 inhibition, caused by neuronal $\mathrm{Zn}^{2+}$ rise. Accordingly, when similar $\mathrm{E}_{\mathrm{GABA}}$ depolarization was obtained blocking $\mathrm{KCC} 2$ with the selective antagonist DIOA ( $20 \mu \mathrm{M} ; 1 \mathrm{~h}$ pre-treatment plus continuous superfusion), acute TPEN application had no effect (Figure 4a).

Using FluoZin-based fluorescence determinations, we also observed that basal intracellular $\mathrm{Zn}^{2+}$ was significantly higher in co-cultured neurons in respect to control (Figure 4b). Neuronal $\mathrm{Zn}^{2+}$ accumulation was prevented by APV/NBQX application in the co-culture medium (Figure 4c), indicating the requirement for GluR activation. Consistently, the application of GCM or glutamate $(20 \mu \mathrm{M})$ onto FluoZin-loaded neurons, elicited an APV/NBQX-sensitive fluorescence increase, rapidly reverting to basal level upon TPEN application (Supplementary Figure S1 and S2).

To identify the source of $\mathrm{Zn}^{2+}$, we performed experiments in the presence of tricine, a chelator of extracellular $\mathrm{Zn}^{2+}$. Tricine treatment $(1 \mathrm{mM}, 24 \mathrm{~h})$ did not abolish the effect of co-culture on $E_{\text {GABA }}$ (Supplementary Table S2), indicating that extracellular $\mathrm{Zn}^{2+}$ is not required for $\mathrm{E}_{\mathrm{GABA}}$ depolarization. However, co-culture induced $\left[\mathrm{Zn}^{2+}\right]_{\mathrm{i}}$ accumulation was not observed (not shown). Noteworthy, tricine treatment caused a reduction in basal $\mathrm{Zn}^{2+}$ both in control and co-cultured neurons ( $n=41 / 59$, control/co-culture; not shown). These results suggest that extracellular $\mathrm{Zn}^{2+}$ chelation modifies neuronal $\mathrm{Zn}^{2+}$ homeostasis, altering basal cytosolic $\mathrm{Zn}^{2+}$ level. To avoid possible effects on intracellular $\mathrm{Zn}^{2+}$ homeostasis, experiments with $\mathrm{Zn}^{2+}$ chelators were repeated reducing incubation time $(4 \mathrm{~h})$, following the observation that $4 \mathrm{~h}$ tricine treatment did not impair neuronal ability to release intracellular $\mathrm{Zn}^{2+}$ in response to a glutamatergic stimulus (Supplementary Figure S2). Consistently, tricine did not prevent the depolarizing effect of $4 \mathrm{~h}$ co-culture on $\mathrm{E}_{\mathrm{GABA}}$ (Figure $4 \mathrm{~d}$ ). Conversely, when intracellular $\mathrm{Zn}^{2+}$ was chelated (with FluoZin $5 \mu \mathrm{M}, 4 \mathrm{~h}$ ), coculture-induced $\mathrm{E}_{\mathrm{GABA}}$ shift was abolished (Figure 4d).

All together, these data indicate that $\mathrm{Cl}^{-}$disequilibrium in co-cultured neurons is due to intracellular $\mathrm{Zn}^{2+}$-dependent KCC2 impairment.

$\mathrm{Zn}^{2+}$-mediated $\mathrm{E}_{\mathrm{GABA}}$ shift requires Src/TrkB activation. To disclose the mechanisms underlying $\mathrm{Zn}^{2+}$-mediated $E_{G A B A}$ shift, neuronal $\left[\mathrm{Zn}^{2+}\right]_{i}$ was artificially increased through perforated (by gramicidin) patch pipette loading. We preliminarily verified the efficacy of intracellular $\mathrm{Zn}^{2+}$ loading through perforated patch by fluorescence recordings; the presence of $\mathrm{ZnCl}_{2}$ in the pipette solution caused a time- and concentration-dependent fluorescence increase in FluoZinloaded neurons, indicating that, in our experimental conditions, $\mathrm{Zn}^{2+}$ permeates through gramicidin pores (Supplementary Table S3; Supplementary Figure S3). When $0.1 \mu \mathrm{M} \mathrm{ZnCl}{ }_{2}$ was added to a BAPTA $(5 \mathrm{mM})$ containing intracellular solution, giving a controlled free $\left[\mathrm{Zn}^{2+}\right]_{i}$ of 
a

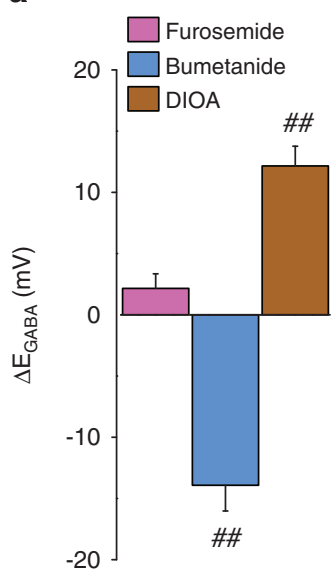

C

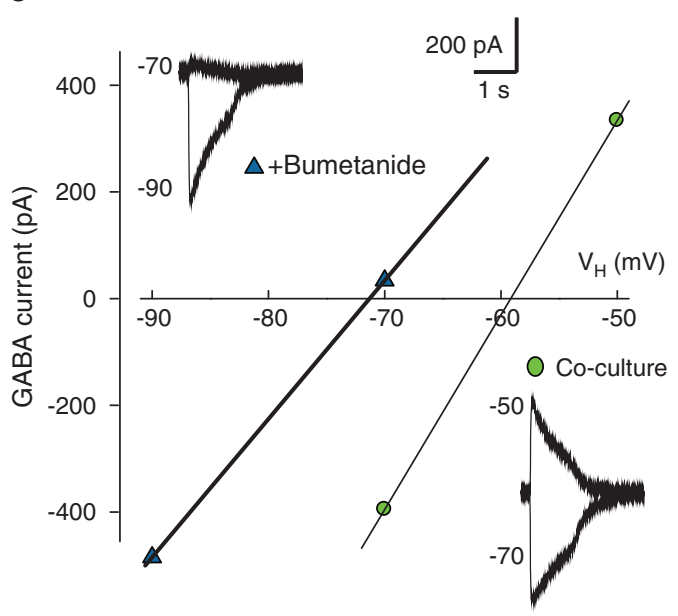

b

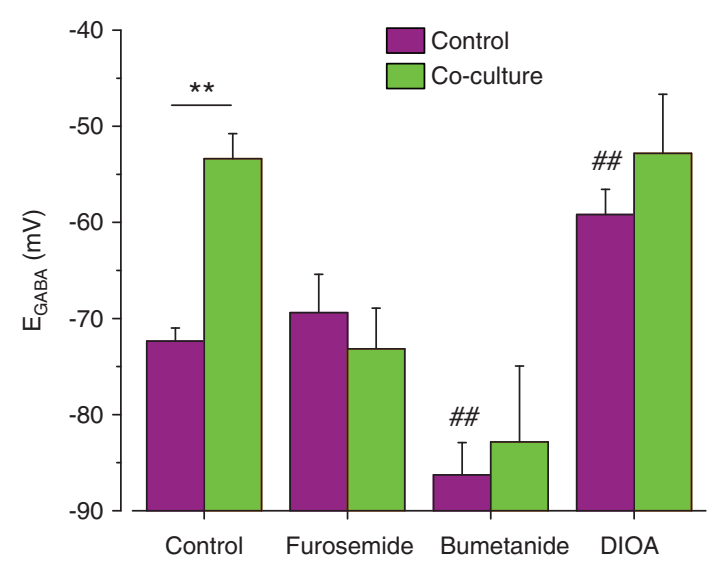

d

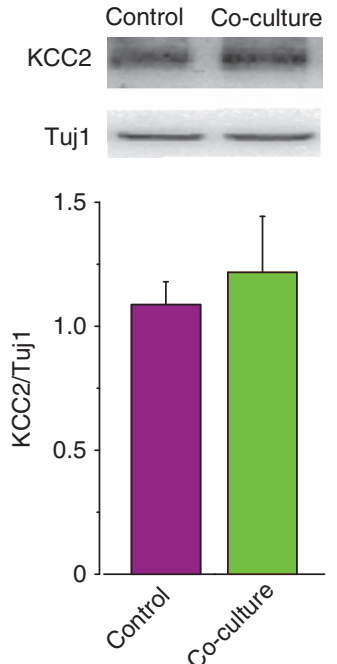

Figure $3 \mathrm{E}_{\mathrm{GABA}}$ depolarizing shift depends on $\mathrm{Cl}^{-}$transporters unbalance. (a) $\mathrm{E}_{\mathrm{GABA}}$ shift in $12 \mathrm{DIV}$ cultured hippocampal neurons induced by acute application of $\mathrm{KCC} 2$ and NKCC1 blocker furosemide (100 $\mu \mathrm{M}, 5-15 \mathrm{~min} ; n=9$; pink), NKCC1 blocker bumetanide (10 $\mu \mathrm{M}, 5-15 \mathrm{~min} ; n=12,{ }^{\# \#} P<0.01$ respect to control; cyan) and KCC2 blocker DIOA (20 $\mu \mathrm{M}, 1 \mathrm{~h}$ pre-application and during the experiment; $n=13,{ }^{\# \#} P<0.01$ respect to control; brown). Notice that furosemide, bumetanide or DIOA application was uneffective on neuronal RMP; (b) $\mathrm{Cl}^{-}$transporters blockers abolish $\mathrm{E}_{\mathrm{GABA}}$ shift induced by glioma co-culture in hippocampal neurons; effect of co-culture on $\mathrm{E}_{\mathrm{GABA}}$ in neurons either untreated (control, $-72.4 \pm 1.4 ; n=14$; co-culture, $-53.4 \pm 2.6 ; n=9 ; P<0.05$ ) or treated with furosemide (control, $-69.4 \pm 4 ; n=9 ;$ co-culture, $-73.2 \pm 4.3 ; n=18 ; P=0.12$ ), bumetanide (control, $-86.3 \pm 3.4 ; n=12 ;$ co-culture, $82.8 \pm 7.9 ; n=9$; ${ }^{\#} P<0.01$ respect to control) and DIOA (control, $-59.2 \pm 2.6 ; n=13 ; c 0$-culture, $-52.8 \pm 6.1 ; n=11$; ${ }^{\# \#} P<0.01$ respect to control) (unpaired $t$-test). (c) Acute bumetanide application reverts $\mathrm{E}_{\mathrm{GABA}}$ shift in co-culture. Traces and $\mathrm{I}-\mathrm{V}$ relation from a single co-cultured neuron before (purple circles) and following application of $10 \mu \mathrm{M}$ bumetanide (cyan triangles; $5-15 \mathrm{~min}, n=5$ ). (d) Glioma co-culture does not alter neuronal KCC2 expression. Bottom, western blot analysis of KCC2 expression in hippocampal neurons in control and after glioma cell co-culture ( $n=6-9$; ANOVA); top, representative immunoblots ofKCC2 (140 KDa) and $\beta$-tubulin III (Tuj1, $60 \mathrm{KDa}$ ) expression in control and co-cultured neurons as indicated

$\sim 10 \mathrm{nM}$ (Supplementary Table S3) $\mathrm{E}_{\mathrm{GABA}}$ promptly shifted to more depolarized values (Figures $5 \mathrm{a}$ and b). Conversely, $\mathrm{E}_{\mathrm{GABA}}$ remained stable with control intracellular solution (calculated as $\sim 0.1 \mathrm{pM}$ free $\left[\mathrm{Zn}^{2+}\right]_{\mathrm{i}}$, Figure $5 \mathrm{a}$ ), or when free $\left[\mathrm{Zn}^{2+}\right]_{\mathrm{i}}$ was below $2 \mathrm{nM}(n=7$; Supplementary Table S3). This time-dependent $E_{\text {GABA }}$ depolarization was not observed when cultures were treated with the KCC2 blocker DIOA $(20 \mu \mathrm{M} ; 1 \mathrm{~h}$ pre-treatment plus continuous superfusion; Figure 5a and Supplementary Table S4). In these conditions, neurons displayed a depolarized $\mathrm{E}_{\mathrm{GABA}}$, likely occluding the effects of intracellular $\mathrm{Zn}^{2+}$ loading. Consistently, acute TPEN application (15 min; Figure $5 \mathrm{~b}$ ) rapidly reverted $\mathrm{Zn}^{2+}$-induced $E_{G A B A}$ depolarization on control neurons. These data indicate that intracellular $\mathrm{Zn}^{2+}$ level rapidly and reversibly interferes with $\mathrm{Cl}^{-}$equilibrium, through $\mathrm{KCC} 2$ activity modulation, highlighting intracellular $\mathrm{Zn}^{2+}$ rise as the key step in gliomainduced $\mathrm{E}_{\mathrm{GABA}}$ depolarization.

Several mechanisms have been proposed to explain KCC2 downregulation in hyperexcitability models, ${ }^{7,20,21}$ including the phosphorylation of KCC2 residues by a number of different kinases. To explore the mechanism of $\mathrm{Zn}^{2+}$-mediated $\mathrm{E}_{\mathrm{GABA}}$ shift, we investigated the involvement of Src/TrkB-dependent KCC2 tyrosine phosphorylation, ${ }^{20,21}$ as intracellular $\mathrm{Zn}^{2+}$ has been reported to transactivate TrkB in a Src-dependent manner. ${ }^{22}$ When hippocampal cultures were treated with TrkB inhibitor K252A (200 nM; $1 \mathrm{~h}$ pre-application and perfused 
a

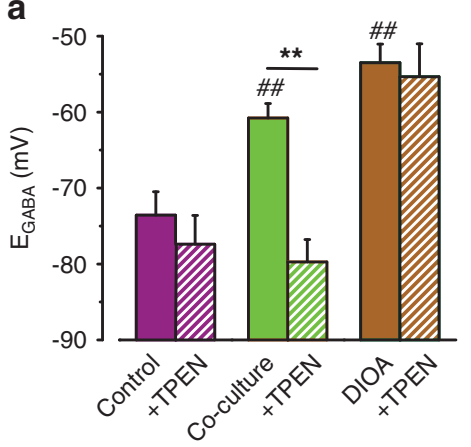

b

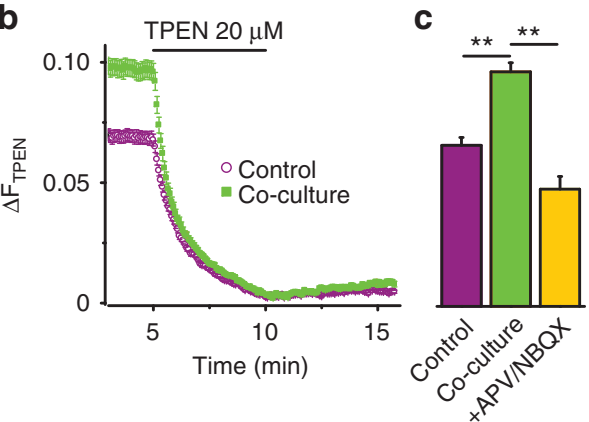

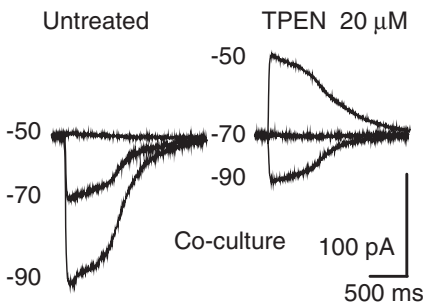

d

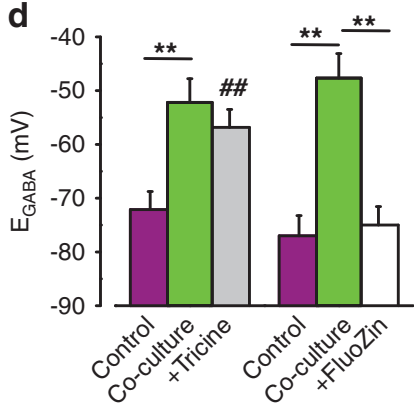

Figure $4 \mathrm{E}_{\mathrm{GABA}}$ shift is dependent on intracellular $\mathrm{Zn}^{2+}$ rise. (a) Co-culture-induced $\mathrm{E}_{\mathrm{GABA}}$ depolarization depends on $\mathrm{Zn}^{2+}$-mediated KCC2 inhibition. Left, TPEN application reverts co-culture induced $\mathrm{E}_{\mathrm{GABA}}$ shift (co-culture/TPEN, $-60.8 \pm 1.9 \mathrm{mV} ;-79.7 \pm 3 \mathrm{mV} ; n=16, P<0.01$; control/TPEN, $-72.8 \pm 1.9 \mathrm{mV} ;-77.4 \pm 3.8 \mathrm{mV} ; n=14 ; P=0.76$; paired $t$-test), but fails to rescue $\mathrm{E}_{\mathrm{GABA}}$ depolarization when $\mathrm{KCC} 2$ is blocked by DIOA (20 $\mu \mathrm{M} ; \mathrm{DIOA}-53.5 \pm 2.4 \mathrm{mV}$; plus TPEN $\left.-55.3 \pm 4.3 \mathrm{mV} ; n=6 ; P=0.78\right)$. Right, traces from a single co-cultured neuron before and following TPEN $(20 \mu \mathrm{M})$ treatment. (b) Co-cultured neurons have higher basal $\mathrm{Zn}^{2+}$. Fluorescence monitoring from FluoZin-3 loaded control (purple circles; $n=125$ ) and co-cultured (green squares; $n=158$ ) neurons exposed to TPEN. (c) Co-culture-induced intracellular $Z n^{2+}$ rise (control, purple column, $n=67$; co-culture, green column, $n=98$ ) is prevented by GluRs blockers (APV $20 \mu \mathrm{M} ; \mathrm{NBQX} 10 \mu \mathrm{M}, n=24$, yellow column ordinate as in $\mathbf{b}$ ). (d) Effects of Zn ${ }^{2+}$ chelation on coculture-induced $\mathrm{E}_{\mathrm{GABA}}$ depolarization. $\mathrm{E}_{\mathrm{GABA}}$ shift was absent when neurons were co-cultured in the presence of the $\mathrm{Zn}^{2+}$-sensitive dye FluoZin $(5 \mu \mathrm{M})$, chelating intracellular $\mathrm{Zn}^{2+}$ (control, $-77 \pm 3.8 \mathrm{mV} ; n=8 ; 4 \mathrm{~h}$ co-culture $-47.7 \pm 4.6 \mathrm{mV} ; n=13$; $4 \mathrm{~h}$ co-culture plus FluoZin $-75 \pm 3.5 \mathrm{mV} ; n=13 ; P<0.01$ ), while tricine (1 mM, added to the co-culture medium, and washed during recordings, to avoid acute KCC2 inhibition mediated by extracellular $\mathrm{Zn}^{2+}$ removal ${ }^{19}$ ) was ineffective (control, $-72.1 \pm 3.4 \mathrm{mV} ; n=13 ; 4 \mathrm{~h}$ co-culture $-52.2 \pm 4.4 \mathrm{mV} ; n=17 ; 4 \mathrm{~h}$ co-culture plus tricine $-56.8 \pm 3.3 \mathrm{mV} ; n=6 ; P=0.65 ;{ }^{\#} P<0.01$ versus control cultures treated with tricine)

during the experiment), intracellular $\mathrm{Zn}^{2+}$ failed to depolarize neuronal $\mathrm{E}_{\mathrm{GABA}}$ (Figure 5c and Supplementary Table S4). Similarly, in the presence of Src kinase inhibitor PP2 $(5 \mu \mathrm{M} ; 1 \mathrm{~h}$ pre-application and perfused during the experiment), $\mathrm{E}_{\mathrm{GABA}}$ shift due to intracellular $\mathrm{Zn}^{2+}$ loading was absent (Figure $5 \mathrm{c}$ and Supplementary Table S4). Thus, $\mathrm{Zn}^{2+}$-induced $\mathrm{E}_{\mathrm{GABA}}$ shift requires the integrity of Src/TrkB pathway.

Consistently, by western blots analysis, we demonstrated that GCM treatment (15 min) significantly increased neuronal Src phosphorylation (Figure 5d). This effect was $\mathrm{Zn}^{2+}$ dependent, as it was prevented by TPEN application $(20 \mu \mathrm{M}$, $15 \mathrm{~min}$ pre-treatment and during $\mathrm{GCM}$ application, $n=6$; $P=0.92$ with respect to TPEN, Figure 5e). Moreover, GCM treatment significantly increased neuronal TrkB phosphorylation (Figure 5f).

Altogether, these data indicate that glioma-released factors might alter neuronal $\mathrm{Cl}^{-}$homeostasis through $\mathrm{Zn}^{2+}$-induced Src/TrkB-mediated KCC2 modulation, as illustrated in Figure 6.

\section{Discussion}

We used co-cultures of hippocampal neurons and glioma cells to unveil the mechanisms of glioma-induced hyperexcitability, reporting that glioma cells depolarize neuronal $\mathrm{E}_{\mathrm{GABA}}$, increasing $\left[\mathrm{Cl}^{-}\right]_{\mathrm{i}}$ and reverting the driving force for GABA- mediated currents. Our results show that $\mathrm{E}_{\mathrm{GABA}}$ depolarization relies on $\mathrm{Zn}^{2+}$-mediated KCC2 functional impairment, disclosing the underlying mechanism: glioma-released glutamate activates neuronal GluRs, causing neuronal intracellular $\mathrm{Zn}^{2+}$ rise which, through Src/TrkB activation, reduces KCC2 activity, leading to intracellular $\left[\mathrm{Cl}^{-}\right]$increase and $\mathrm{E}_{\mathrm{GABA}}$ depolarization. We conclude that glioma might reduce neuronal inhibition through $\mathrm{Zn}^{2+}$-mediated downregulation of KCC2 activity, causing hyperexcitability.

In glioma-co-cultured hippocampal neurons, the currentvoltage relationship of GABA-mediated responses is shifted to more depolarized potentials, compared with control, giving a more depolarized $\mathrm{E}_{\mathrm{GABA}}$. This indicates a higher basal neuronal $\left[\mathrm{Cl}^{-}\right]_{\mathrm{i}}$ in co-cultures, confirmed by an independent estimation in neurons transfected with a YFP-based $\mathrm{Cl}^{-}$Sensor. Although glioma co-culture induces a small neuronal depolarization, the shift of $\mathrm{E}_{\mathrm{GABA}}$ is more relevant, resulting in the inversion of the driving force for GABA-mediated currents.

According to previous studies, $\mathrm{Cl}^{-}$homeostasis in cultured hippocampal neurons is determined by the activity of both NKCC1 and KCC2. ${ }^{8}$ Indeed, we show here that both transporters are expressed in control neurons and their activity is required to maintain $\mathrm{Cl}^{-}$equilibrium as blocking either NKCC1 or KCC2 leads to a shift in basal $\mathrm{E}_{\mathrm{GABA}}$. Conversely, in co-cultured neurons, NKCC1 activity is 

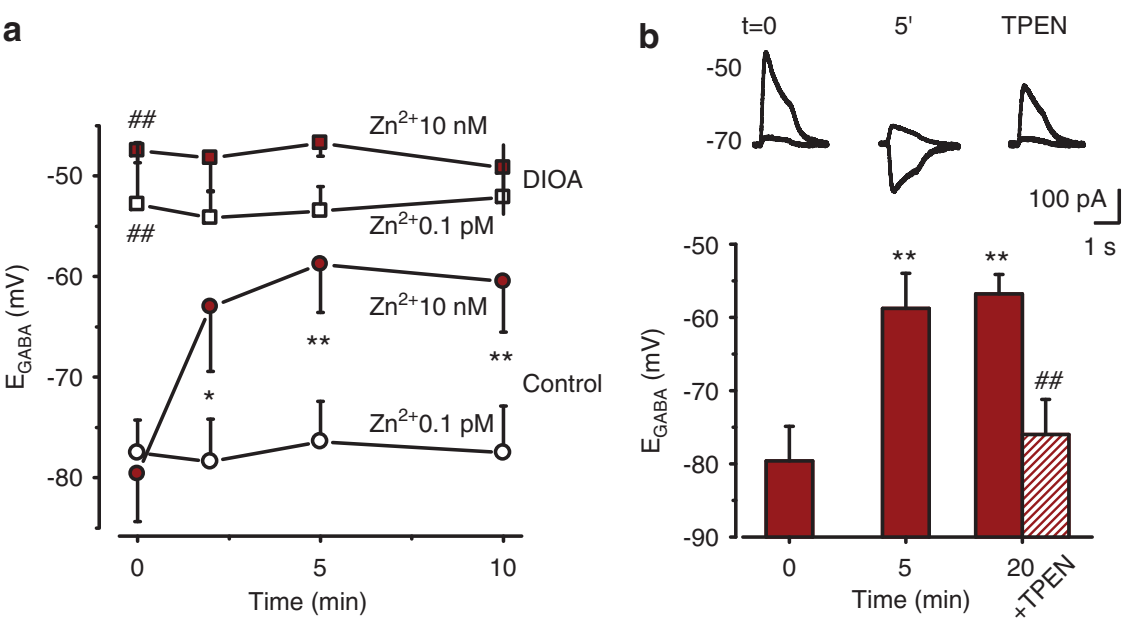

C

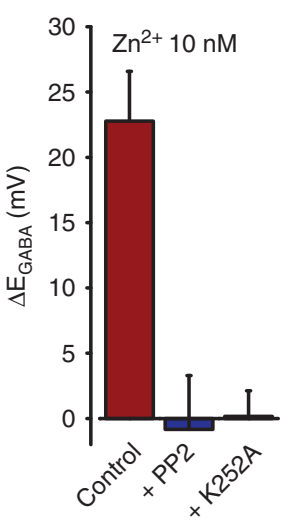

d
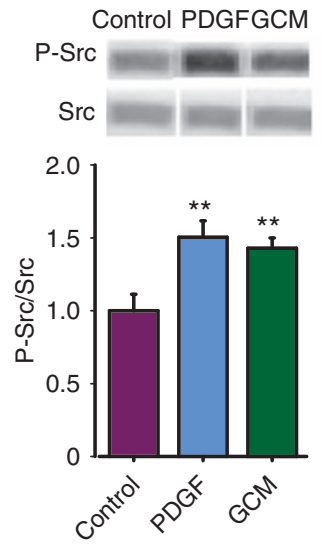

e

Control GCM

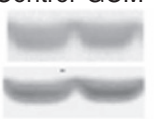

TPEN $10 \mu \mathrm{M}$

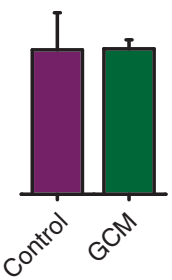

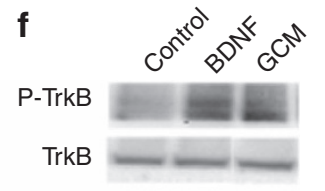

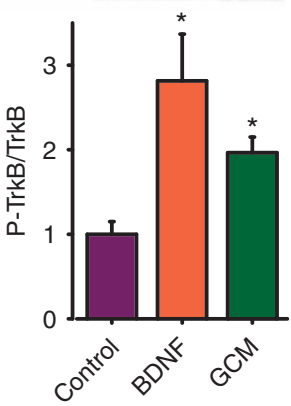

Figure 5 Intracellular $\mathrm{Zn}^{2+}$ modulates $\mathrm{E}_{\mathrm{GABA}}$ through $\mathrm{Src}$ and TrkB activation. (a) Bottom, $\mathrm{E}_{\mathrm{GABA}}$ is rapidly depolarized in cultured hippocampal neurons loaded with $\sim 10 \mathrm{nM}$ free $\mathrm{Zn}^{2+}$ through the patch pipette (dark red circles; $t=0,-79.6 \pm 4.7 \mathrm{mV} ; \mathrm{t}=5^{\prime},-58.8 \pm 4.8 \mathrm{mV} ; n=17 ;{ }^{*} P<0.05 ;{ }^{* \star} P<0.01$, paired $t$-test), but not with standard EGTAcontaining intracellular solution (empty circles; free $\left.\mathrm{Zn}^{2+}=0.1 \mathrm{pM} ; P=0.54 ; n=8\right)$. Top, in cultures treated with the KCC2 blocker DIOA (20 $\mu \mathrm{M}, 1 \mathrm{~h}$ pre-application and during the experiment), $\mathrm{E}_{\mathrm{GABA}}$ was significantly depolarized at $t=0$ with both intracellular solutions (dark red squares, free $\mathrm{Zn}^{2} \sim 10 \mathrm{nM},-47.5 \pm 1.2 \mathrm{mV} ; n=5$; empty squares, free $\mathrm{Zn}^{2+}=0.1 \mathrm{pM},-52.8 \pm 6.1 \mathrm{mV}, n=6$; ${ }^{\# \#} P<0.01$; respect to control, ANOVA ), and remained stable despite the intracellular $\mathrm{Zn}^{2+}$ loading. (b) TPEN application reverts $\mathrm{Zn}{ }^{2+}$ induced $\mathrm{E}_{\mathrm{GABA}}$ shift. Top, sample current traces from $10 \mathrm{nM}$ free $\mathrm{Zn}^{2+}$-loaded neuron, at start of recording $(t=0), 5^{\prime}$ after and following TPEN application (20 $\left.\mu \mathrm{M} ; 15^{\prime}\right)$. Bottom, $\mathrm{Zn}^{2+}$-induced $\mathrm{E}_{\mathrm{GABA}}$ depolarization at $t=0 ; 5^{\prime}$ and $20^{\prime}\left(n=7,{ }^{* *} \mathrm{P}<0.01\right.$, ANOVA); dashed column represents $\mathrm{E}_{\mathrm{GABA}}$ after $15^{\prime}$ TPEN application (following $5^{\prime} \mathrm{Zn}^{2+}$ loading; $n=8,{ }^{\#} P<0.01$ versus $20^{\prime}$, ANOVA-Tukey test). (c) Impairment of $Z n^{2+}$-induced $E_{G A B A}$ depolarization $\left(\Delta \mathrm{E}_{\mathrm{GABA}}\right.$ at $\left.\mathrm{t}=5^{\prime}, 22.8 \pm 3.8 \mathrm{mV}, n=9\right)$ in the presence of Src kinases inhibitor (PP2, $5 \mu \mathrm{M}, n=9, P<0.01$ ) or TrkB inhibitor (K252A, $200 \mathrm{nM} ; n=7, P<0.01$ ) (versus control, ANOVA). $\mathrm{E}_{\mathrm{GABA}}$ and RMP were depolarized in control following, K252A or PP2 treatment as reported in Supplementary Table S4. (d) GCM increases Src phosphorylation. Top, typical immunoblot experiment; bottom, GCM treatment (15') increases Src $(n=10, P<0.01$; ANOVA) phosphorylation (platelet-derived growth factor represent positive control). (e) TPEN application prevents GCM-induced Src phosphorylation; Top, typical immunoblot experiment; bottom, GCM treatment (15') failed to increase Src $\left(n=6, P=0.92\right.$; ANOVA) phosphorylation in cultures pre-treated with TPEN $\left(20 \mu \mathrm{M}, 15^{\prime}\right.$; $y$-axis as in (d). (f) GCM increases TrkB phosphorylation. Top, typical immunoblot experiment; bottom, GCM treatment $\left(15^{\prime}\right)$ increases TrkB $(n=3, P<0.05 ;$ ANOVA) phosphorylation (BDNF represents positive control)

apparently not balanced by $\mathrm{KCC}$-mediated $\mathrm{Cl}^{-}$extrusion and transporter disequilibrium leads to an increase in neuronal $\left[\mathrm{Cl}^{-}\right]_{\mathrm{i}}$. This picture is demonstrated pharmacologically by the use of selective antagonists of $\mathrm{Cl}^{-}$co-transporters: (i) furosemide, the blocker of both transporters, abolishes $E_{G A B A}$ shift, demonstrating the involvement of $\mathrm{Cl}^{-}$transport; (ii) the specific NKCC1 antagonist bumetanide reverts coculture-induced $E_{G A B A}$ depolarization, demonstrating that $\mathrm{Cl}^{-}$ accumulation requires the activity of NKCC1; (iii) neurons treated with the specific KCC2 blocker DIOA display depolarized $E_{G A B A}$, likely occluding the co-culture effect. Altogether, these data support the proposal that in neurons, a new $\mathrm{Cl}^{-}$ equilibrium is established during glioma co-culture, characterized by higher $\left[\mathrm{Cl}^{-}\right]_{\mathrm{i}}$ and caused by reduced KCC2 activity.

We provide evidence that glioma-induced KCC2 impairment, observed in co-cultured neurons, is due to intracellular $\mathrm{Zn}^{2+}$ rise. $^{18}$ Indeed, intracellular $\mathrm{Zn}^{2+}$ chelation by TPEN rapidly reverts co-culture-induced $\mathrm{E}_{\mathrm{GABA}}$ shift and basal $\left[\mathrm{Zn}^{2+}\right]_{\mathrm{i}}$ is significantly higher in neurons after glioma co-culture.

The effect of neuronal $\mathrm{Zn}^{2+}$ rise on $\mathrm{E}_{\mathrm{GABA}}$ was directly evaluated in experiments where intracellular $\left[\mathrm{Zn}^{2+}\right]$ was artificially increased from pico to nanomolar, as in pathological conditions. $^{23,24}$ The resulting $\mathrm{Zn}^{2+}$-dependent $\mathrm{E}_{\mathrm{GABA}}$ shift is ascribable to KCC2 impairment, because it was absent when 


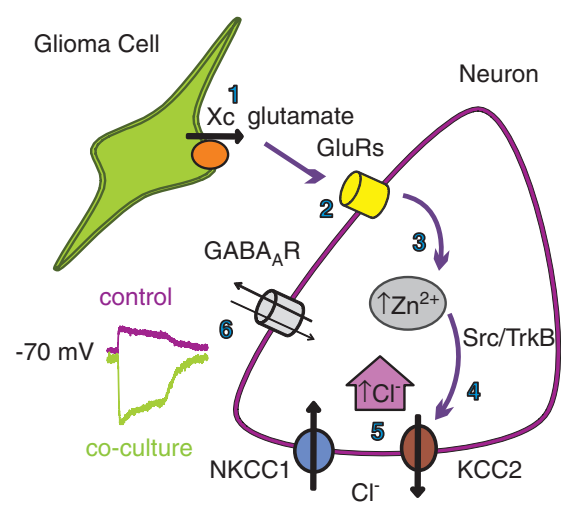

Figure 6 Model of glioma-induced $\mathrm{E}_{\mathrm{GABA}}$ shift. Glutamate released from glioma cells (1) causes GluR-dependent (2) neuronal [ $\left.\mathrm{Zn}^{2+}\right]_{\mathrm{i}}$ increase (3), Src/TrkB-mediated $\mathrm{KCC} 2$ inactivation (4) leading to $\left[\mathrm{Cl}^{-}\right]$increase (5) and $\mathrm{E}_{\mathrm{GABA}}$ shift (6)

KCC2 was pharmacologically blocked by DIOA. Indeed, the effect of $\mathrm{Zn}^{2+}$ on $\mathrm{E}_{\mathrm{GABA}}$ is likely occluded by pharmacological KCC2 block, as DIOA treated neurons already show a depolarized

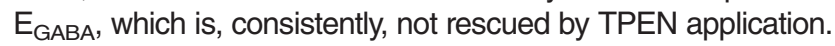

Glioma-induced alteration of neuronal $\mathrm{Cl}^{-}$homeostasis likely depends on functional KCC2 block, rather than on reduction of protein expression (see Lee et al. ${ }^{7}$ ). This view is based on the unaltered expression of neuronal KCC2 after glioma co-culture and on the rapid rescue exerted by $\mathrm{Zn}^{2+}$ chelation, demonstrating a dynamic modulation of $\mathrm{Cl}^{-}$transport mechanisms. The functional modulation of KCC2 activity has been observed both in physiological and pathological models, such as prolonged post-synaptic spiking, ${ }^{25}$ brainderived neurotrophic factor (BDNF) stimulation ${ }^{26}$ and oxygen glucose deprivation. ${ }^{18}$ Our data suggest that the mechanism responsible for glioma-induced $\mathrm{KCC} 2$ inhibition relies on $\mathrm{Zn}^{2+}$ mediated Src/TrkB activation. Indeed, $\mathrm{Zn}^{2+}$-induced $\mathrm{E}_{\mathrm{GABA}}$ shift was prevented by the application of Src or TrkB kinase inhibitors (PP2 or K252A). Consistently, GCM increased the level of TrkB and Src phosphorylation in hippocampal cultures, the latter effect being prevented by the $\mathrm{Zn}^{2+}$ chelator TPEN. These results are in line with the notion that intracellular $\mathrm{Zn}^{2+}$ may transactivate TrkB by a neurotrophin-independent and Src-dependent mechanism, as reported in models of intense neuronal activity. ${ }^{22}$

In neurons, $\mathrm{Zn}^{2+}$-induced TrkB transactivation may mimic BDNF-TrkB signaling, leading to KCC2 phosphorylation on tyrosine residues ${ }^{27,28}$ and driving neuronal disinhibition. ${ }^{29} \mathrm{We}$ can speculate that co-culture induced $\mathrm{E}_{\mathrm{GABA}}$ depolarization needs KCC2 phosphorylation on tyrosine residues because of $\mathrm{Zn}^{2+}$-induced TrkB transactivation.

We report that glioma-induced $E_{\mathrm{GABA}}$ shift requires the release of glutamate in the extracellular space by glioma cells and the consequent activation of neuronal ionotropic GluRs. Indeed, the application of APV and NBQX during co-culture prevents $\mathrm{E}_{\mathrm{GABA}}$ depolarization.

It is known that glutamate can induce intracellular $\mathrm{Zn}^{2+}$ increase through different mechanisms, including AMPARs and $\mathrm{Ca}^{2+}$ channel-mediated influx or $\mathrm{Ca}^{2+}$-dependent intracellular release. ${ }^{25,30}$ We observed that both APV and NBQX abolished co-culture-induced $\mathrm{E}_{\mathrm{GABA}}$ depolarization highlighting the role for both ionotropic GluRs in this effect. The simplest explanation for this evidence is that AMPARmediated neuronal depolarization drives NMDAR activation thus allowing $\mathrm{Ca}^{2+}$-dependent intracellular $\mathrm{Zn}^{2+}$ release. ${ }^{30}$ Our data indicate that the source of $\mathrm{Zn}^{2+}$ is intracellular, as the extracellular $\mathrm{Zn}^{2+}$ chelator tricine was ineffective, whereas FluoZin prevented co-culture-induced $\mathrm{E}_{\mathrm{GABA}}$ depolarization.

It has to be considered that tricine treatment, although did not prevent co-culture-induced $\mathrm{E}_{\mathrm{GABA}}$ depolarization, inhibited co-culture-induced basal $\left[\mathrm{Zn}^{2+}\right]_{\mathrm{i}}$ accumulation. Thus, perturbing extracellular $\mathrm{Zn}^{2+}$ concentration may modify neuronal $\mathrm{Zn}^{2+}$ homeostasis, preventing cytosolic $\mathrm{Zn}^{2+}$ accumulation. However, tricine-treated neurons retained the ability to release intracellular $\mathrm{Zn}^{2+}$ in response to a glutamatergic stimulus, and this event is likely sufficient to trigger the intracellular signaling leading to KCC2 impairment.

It is well established that intracellular $\mathrm{Zn}^{2+}$ rise induces cell death, and $\mathrm{Zn}^{2+}$ exposure is toxic to neurons both in vitro and in vivo. It is now evident that increased cytosolic $\mathrm{Zn}^{2+}$ resulting from liberation from intracellular stores, rather than cytoplasmic influx of synaptically released $\mathrm{Zn}^{2+}$, can be highly toxic during oxidative and other types of neuronal injury, ${ }^{31}$ and $\mathrm{Zn}^{2+}$ dyshomeostasis appears to be a common feature of numerous neuropathological conditions. ${ }^{23,32}$ We speculate that the reported mechanism, leading to reduced GABAergic transmission, could underlie the etiology of glioma-related epilepsy, pointing to $\mathrm{Zn}^{2+}$ accumulation as a possible therapeutic target to restore KCC2 function and the excitatory/inhibitory balance. In this view, it is possible to speculate that $\mathrm{Zn}^{2+}$ homeostatic drugs may be helpful in the treatment of $\mathrm{Zn}^{2+}$-related neurological disorders such as neuronal hyperexcitability or Alzheimer's disease. ${ }^{33}$

The use of co-cultures ${ }^{34}$ allowed to disclose the molecular mechanisms involved in glutamate-mediated overexcitation induced by glioma. Our results are in line with recent works showing that $\mathrm{Xc}$-mediated glutamate release is responsible for the generation of tumor-associated epileptic events in gliomabearing mice. ${ }^{6,35}$ Indeed, increased concentration of extracellular glutamate has been found in peritumoral tissue in both humans and mice, ${ }^{6,36}$ supporting its role in tumor growth, survival and peritumoral seizure activity. ${ }^{6}$ Additional sources of glutamate in peritumoral tissue may be microglial Xc system or reverse activity of $\mathrm{Na}^{+}$-dependent glutamate transporters in neurons or astrocytes. ${ }^{37,38}$ Thus, the co-culture system likely retains the feature of excessive glutamate release typical of glioma. Consistently, neuronal $\mathrm{E}_{\mathrm{GABA}}$ shift was observed in coculturing neurons with different glioblastoma cell lines, but not with astrocytes, supporting the view of a tumor-specific effect. ${ }^{6}$

Glutamate-induced alteration of neuronal $\mathrm{Cl}^{-}$homeostasis may act concomitantly with other mechanisms, including the direct depolarizing effect of glutamate on neurons, displacing the excitation/inhibition balance toward an increased network excitability, thus promoting seizure onset. ${ }^{35}$

In conclusion, our study provides a possible explanation of the mechanisms by which glioma cells affect neuronal $\mathrm{Cl}^{-}$ equilibrium, highlighting the role of $\mathrm{Zn}^{2+}$, recently emerged in a variety of excitotoxic conditions, such as epilepsy, ischemia and brain trauma. ${ }^{32}$ 
Materials and Methods

Animals. Procedures using laboratory animals were in accordance with the international guidelines on the ethical use of animals from the European Communities Council Directive of November 24, 1986 (86/609/EEC). All efforts were made to minimize animal suffering and to reduce the number of animals used in accordance with the European Communities Council Directive of September 20, 2010 (2010/63/UE).

Primary hippocampal neuronal cultures. Hippocampal neuronal cultures were prepared from newborn (P0-P1) C57BL/6 mice of either sex (Charles River - Research Models and Services, Lecco, Italy). In brief, after careful dissection from diencephalic structures, the meninges were removed and the hippocampi were chopped and digested in $1.25 \mathrm{mg} / \mathrm{ml}$ trypsin for $20 \mathrm{~min}$ at $37^{\circ} \mathrm{C}$. Cells were mechanically dissociated and plated at a density of $10^{5}$ in poly-L-lysine-coated glass coverslip (12 mm diameter) in serum-free Neurobasal Medium (Gibco Life Science, Life Technologies Italia, Monza, Italy), supplemented with B27 2 mM L-glutamine and $100 \mu \mathrm{g} / \mathrm{ml}$ gentamicin (neuronal culture medium). Then, cells were kept at $37^{\circ} \mathrm{C}$ in $5 \% \mathrm{CO}_{2}$ for $10-13$ days with medium replacement (1:1 ratio) three times per week. With this method, we obtained cultures composed by $60-70 \%$ neurons, $30-35 \%$ astrocytes and $4-5 \%$ microglia, as determined with $\beta$-tubulin III, glial fibrillary acidic protein and isolectin IB4 staining. ${ }^{38}$ The same procedure was followed to prepare rat hippocampal culture used for some immunoblot experiments.

Glial primary cultures. Primary cortical glial cells were prepared from P0-P2 mice. Cerebral cortices were chopped and digested in $30 \mathrm{U} / \mathrm{ml}$ papain for $40 \mathrm{~min}$ at $37^{\circ} \mathrm{C}$ and gently triturated. The dissociated cells were washed, suspended in Dulbecco's Modified Eagle Medium (Gibco, Life Technologies Italia) with Glutamax with 10\% fetal bovine serum (Invitrogen, Life Technologies Italia) and plated at a density of $9-10 \times 10^{5}$ in $175 \mathrm{~cm}^{2}$ cell culture flasks. At confluence (10-14 days in vitro, DIV), glial cells were shaken for $2 \mathrm{~h}$ at $37^{\circ} \mathrm{C}$ to detach and remove microglial cells. These procedures gave almost pure astrocytes cell population (4-6\% of microglia contamination), as verified by staining with glial fibrillary acidic protein and isolectin IB4. ${ }^{34}$

Neuronal culture transfection. Hippocampal neuronal cultures were transfected at 9-10 DIV. One day before transfection, $50 \%$ of the culture medium was replaced with fresh medium. For transfection, $100 \mu \mathrm{l}$ of Neurobasal media were mixed with $2 \mu \mathrm{l}$ of NeuroMag (OZ Bioscience, Marseille, France) and $1 \mu \mathrm{g}$ of ClSensor cDNA. ${ }^{15,16}$ The mixture was incubated for $15-20 \mathrm{~min}$ at room temperature and thereafter distributed dropwise over the neuronal culture. Neuronal cultures were placed on a magnetic board (OZ Bioscience) and incubated for $15 \mathrm{~min}\left(37^{\circ} \mathrm{C}\right.$, $5 \% \mathrm{CO}_{2}$ ). One hour later, $50 \%$ of neuronal culture medium was substituted with fresh neuronal culture medium. Cells were used for experiments $24-76 \mathrm{~h}$ after transfection.

Glioma cell culture. Patient-derived glioma cells (MZC; kindly provided by Dr. Antonietta Arcella, Neuromed, Italy) within passage $40,{ }^{14,39}$ glioblastoma cell lines: GL15, ${ }^{14,40}$ U87MG (American Type Culture Collection, Rochville, MD, USA) and GL261 ${ }^{41}$ were grown in Dulbecco's Modified Eagle Medium with Glutamax supplemented with $10 \%$ heat-inactivated fetal bovine serum, $100 \mathrm{U} / \mathrm{ml}$ penicillin $\mathrm{G}$, $2.5 \mu \mathrm{g} / \mathrm{ml}$ amphotericin B and $100 \mu \mathrm{g} / \mathrm{ml}$ streptomycin at $37^{\circ} \mathrm{C}$ in a $5 \% \mathrm{CO}_{2}$ humidified atmosphere. Medium was changed three times per week; cells were used between 20th and 40th passage.

Co-cultures. Glioma cells or astrocytes, prepared as above, were re-suspended in neuronal culture medium and replated by seeding $10^{5}$ cells onto $0.33 \mathrm{~cm}^{2}$ transwell cell-culture inserts (pore size $0.4 \mu \mathrm{M}$, Corning B.V. Life Sciences, Amsterdam, The Netherlands), allowing traffic of small diffusible substances, but preventing cell-to-cell contact. Transwell inserts were transferred into 24-well cultures plates, containing 10-12 DIV primary hippocampal cultures in neuronal culture medium.

Neuronal viability after 4 and $24 \mathrm{~h}$ of co-culture was evaluated as reported in Supplementary Figure S4.

Patch-clamp recordings. Patch-clamp recordings were obtained using glass electrodes (3-5 M $\Omega$ ) filled with the following intracellular solution (in $\mathrm{mM}$ ): $140 \mathrm{KCl}$, $2 \mathrm{MgCl}_{2}, 10 \mathrm{HEPES}, 2 \mathrm{MgATP}, 0.5 \mathrm{EGTA}$; $\mathrm{pH} 7.3$, with $\mathrm{KOH}$. Perforated patch-clamp recordings with access resistances between 30 and $40 \mathrm{M} \Omega$ were obtained using gramicidin $\mathrm{D} .{ }^{42}$ Gramicidin, prepared every $2 \mathrm{~h}$, was added to the pipette solution to a final concentration of $50 \mu \mathrm{g} / \mathrm{ml}$. During experiments, neurons were continuously superfused with normal extracellular solution containing (in $\mathrm{mM}$ ): $140 \mathrm{NaCl}, 2.5 \mathrm{KCl}$, $2 \mathrm{CaCl}_{2}, 2 \mathrm{MgCl}_{2}, 10$ HEPES-NaOH and 10 glucose (pH 7.3), added with tetrodotoxin $(0.2 \mu \mathrm{M})$, using a gravity-driven perfusion system, consisting of independent tubes for standard and agonist-containing solutions, connected to a fast exchanger system (RSC-100; Bio-Logic, Claix, France). All recordings were performed at $24-25^{\circ} \mathrm{C}$. For I-V experiments, neurons were voltage-clamped at $-70 \mathrm{mV}$ with 6 -s steps to each test potential; GABA ( $100 \mu \mathrm{M} ; 500 \mathrm{~ms}$; every $30 \mathrm{~s}$ ) was applied $1 \mathrm{~s}$ after the voltage step onset. A linear regression was used to calculate the voltage dependence of GABA-evoked current. $\mathrm{E}_{\mathrm{GABA}}$ was taken as the intercept of this best-fit line for each cell. We minimized bicarbonate flux through the $\mathrm{GABA}_{A}$ channels, using an HEPES-buffered extracellular solution (nominally $\mathrm{CO}_{2}-$ and bicarbonate-free ${ }^{43,44}$ ), so that $E_{\text {GABA }}$ was an estimation of $E_{C l}$. Membrane currents, recorded with a patch-clamp amplifier (Axopatch 200B; Molecular Devices, Foster city, CA, USA), were filtered at $2 \mathrm{kHz}$, digitized $(10 \mathrm{kHz})$ and acquired with Clampex 10 software (Molecular Devices). The stability of the patch was checked by repetitively monitoring the input and series resistance during the experiment, and recordings were discarded when any of these parameters changed by $>10 \%$.

Zn2+ loading through gramicidin pores. When indicated (Figure 5 and Supplementary Figure S3), intracellular $\left[\mathrm{Zn}^{2+}\right]$ was artificially increased in neurons through the perforated patch, using a BAPTA-based intracellular solution (in $\mathrm{mM}$ : $140 \mathrm{KCl}, 2 \mathrm{MgCl}_{2}$, 10 HEPES, 2 MgATP, 5 BAPTA) supplemented with $\mathrm{ZnCl}_{2}(0.01-$ $100 \mu \mathrm{M})$. The corresponding free $\mathrm{Zn}^{2+}$ concentrations were calculated using the MaxChelator software: http://www.stanford.edu/ cpatton/maxc.html, and are reported in Supplementary Table S3.

$\mathrm{Zn}^{+2}$ imaging. Fluorescence images were acquired at room temperature $\left(24-25^{\circ} \mathrm{C}\right)$ using a customized digital imaging microscope. Excitation of fluorophores at various wavelengths was achieved using a 1-nm-bandwidth polychromatic light selector (Till Polychrome V), equipped with a $150 \mathrm{~W}$ xenon lamp (Till Photonics, Hillsboro, OR, USA). Fluorescence was visualized using an upright microscope (Axioskope) equipped with a 40x water-immersion objective (Achroplan CarlZeiss, Oberkochen, Germany) and a digital 12 bit CCD camera system (SensiCam, PCO AG, Kelheim, Germany). All the peripheral hardware control, image acquisition and image processing were achieved using customized software Till Vision v. 4.0 (Till Photonics). Changes in the intracellular $\mathrm{Zn}^{2+}$ level were monitored using the high-affinity $\mathrm{Zn}^{2+}$-sensitive indicator FluoZin-3AM (Invitrogen). Neurons were loaded by incubating coverslips for $45 \mathrm{~min}$ at $37^{\circ} \mathrm{C}$ in $1 \mathrm{ml}$ of HEPESbuffered salt solution containing: $120 \mathrm{mM} \mathrm{NaCl}, 5.4 \mathrm{mM} \mathrm{KCl}, 1.8 \mathrm{mM} \mathrm{CaCl}_{2}, 0.8 \mathrm{mM}$ $\mathrm{MgCl}_{2}, 20 \mathrm{mM}$ HEPES-NaOH and $15 \mathrm{mM}$ glucose $(\mathrm{pH} 7.3)$, plus $5 \mathrm{mg} / \mathrm{ml}$ of bovine serum albumin and $5 \mu \mathrm{M}$ of FluoZin-3AM. For time-lapse recordings, FluoZin-3AM was excited at $480 \mathrm{~nm}$ (emission filter D535/40 nm, dichroic mirror 505DCLP). $\mathrm{Zn}^{2+}$ fluorescence changes are presented as $\Delta \mathrm{F} / \mathrm{F}_{0}=\left(\mathrm{F}-\mathrm{F}_{0}\right) / \mathrm{F}_{0}$, or $\Delta \mathrm{F}_{\mathrm{TPEN}}=$ $\left(F_{0}-F_{\text {TPEN }}\right) / F_{\text {TPEN }}$, where $F$ is the current fluorescence intensity, $F_{0}$ is the fluorescence intensity before agonist application and $\mathrm{F}_{T P E N}$ represents fluorescence intensity after $5 \mathrm{~min}$ of TPEN application.

$\mathrm{Cl}^{-}$imaging. For non-invasive monitoring of $\left[\mathrm{Cl}^{-}\right]_{\text {, }}$, cells were transfected with CFP/YFP-based Cl-Sensor ${ }^{15}$ and the optic system equipped with an emission filter D535/40 nm and a dichroic mirror 505DCLP $(505 \mathrm{~nm})$ was used. Cells expressing Cl-Sensor were excited at 445 and $485 \mathrm{~nm}$ wavelengths alternatively $(50 \mathrm{~ms}$, $0.1 \mathrm{~Hz}$ ). $\left[\mathrm{Cl}^{-}\right]$, changes are expressed as a ratio $(\mathrm{R})$ of background-subtracted $\mathrm{F} 445$ and F485 ( $R=F 445 / F 485)$. (Supplementary Figure S5) Calibration of Cl-Sensor (Supplementary Figure S6) was performed as in Markova et al. ${ }^{15}$ and Wassem et al. ${ }^{16}$ External solutions with different $\mathrm{Cl}^{-}$concentrations (in $\mathrm{mM}: 1,3,7,12,20$, $35,50,70,82.4,130,164.8$ ) were obtained by mixing the following solutions (in $\mathrm{mM}$ ): (i) $164.8 \mathrm{KCl}, 10 \mathrm{D}$-glucose, $20 \mathrm{HEPES}, \mathrm{pH} 7.32$ and (ii) $164.8 \mathrm{~K}$-gluconate, 10 D-glucose, 20 HEPES, pH 7.32. To increase the permeability of the cell membrane to $\mathrm{Cl}^{-}$ions, $\beta$-escin ( $80 \mu \mathrm{M}$, Sigma, St Louis, MO, USA) was added to the extracellular solution and applied during 2-5 min. Fluorescence signals corresponding to rising $\mathrm{Cl}^{-}$concentrations were monitored from single $\mathrm{Cl}$-Sensor expressing neurons, after $\beta$-escin removal.

Glioma-conditioned medium (GCM). GCM was prepared as follows: when cultures of glioma cells became confluent $\left(\sim 3 \times 10^{6}\right.$ in $10 \mathrm{~cm}$ culture dishes), the culture medium was substituted with $10 \mathrm{ml}$ of filtered normal extracellular solution. The conditioned medium (GCM) was collected after incubating for $4 \mathrm{~h}$, centrifuged, pH adjusted to 7.35 and then used for electrophysiological and imaging 
experiments. ${ }^{17}$ For immunoblot analysis of TrkB and Src activation, GCM was obtained incubating $(4 \mathrm{~h})$ glioma cells in Locke's buffer (in mM: $154 \mathrm{NaCl}, 5.6 \mathrm{KCl}$ 3.6 $\mathrm{NaHCO}_{3}, 2.3 \mathrm{CaCl}_{2}, 1 \mathrm{MgCl}_{2}$, 5.6 glucose, 5 HEPES; pH 7.4).

Cell stimulation and western blot analysis. For western blot experiments, $4 \times 10^{5}$ hippocampal cells were plated on poly-L-lysine coated 12-well cultures plates. ${ }^{45}$

For the analysis of TrkB and Src activation, cultured hippocampal neurons were incubated for $2 \mathrm{~h}$ in Locke's buffer and stimulated for $15 \mathrm{~min}$ with either GCM or drugs: BDNF $(100 \mathrm{ng} / \mathrm{ml})$, platelet-derived growth factor $(100 \mathrm{ng} / \mathrm{ml})$. To assess the role of $\mathrm{Zn}^{2+}$ rise in Src phosphorylation, hippocampal cultures were treated with the $\mathrm{Zn}^{2+}$ chelator TPEN (20 $\mu \mathrm{M}, 15 \mathrm{~min}$ pre-application, and during GCM stimulation). Corresponding cell lysates were run on SDS-polyacrylamide gel and analyzed for Src or TrkB phosphorylation. Densitometric analysis was performed with QuantityOne software (Bio-Rad Laboratories S.r.l., Segrate (MI), Italy) and phosphoprotein levels were normalized for TrkB and Src expression. For KCC2 expression determination, western blot analysis was performed on rat hippocampal cell lysates in control conditions and after $24 \mathrm{~h}$ co-culture with equal number of glioma cells. For each condition, equal amounts of proteins were loaded on SDS-PAGE gel for immunoblot analysis with rabbit anti-KCC2. Protein levels were normalized for TuJ1 expression.

Drugs. GABA, Bicuculline methochloride, Tetrodotoxin citrate, NBQX and D-APV were purchased from Abcam Biochemicals (Cambridge, UK); Furosemide (100 mM in dimethyl sulfoxide (DMSO)), Bumetanide (50 mM in EtOH) and PP2 (10 mM in DMSO) were purchased from Tocris Biosciences (Bristol, UK); FluoZin-3AM (Invitrogen, 5 mM DMSO); K252a (Calbiochem, Merck S.p.a., Milano, Italy); DIOA (Santa Cruz Biotechnology, Inc., Heidelberg, Germany; $200 \mathrm{mM}$ in EtOH); BDNF (PeproTech EC Ltd., London, UK); TPEN (10 mM in EtOH), Gramicidin (50 mg/ml in DMSO), Sulfasalazine (500 mM in DMSO), platelet-derived growth factor and al chemicals used were purchased from Sigma-Aldrich, Milan, Italy. Where not indicated, all drugs were dissolved in water. Antibodies were purchased and used as follows: rabbit anti-Phospho TrkB (phospho Y515, 1: 500, Abcam, Cambridge, UK), rabbit anti-Phospho Src (phospho Y416, 1:2000, Abcam), mouse anti- Neuronal Class III $\beta$-tubulin (TuJ1, 1 : 2000, n.cat MMS-435P, Covance, San Diego, CA, USA), rabbit anti-actin (1:5000, Sigma-Aldrich); rabbit anti-KCC2 (1:1000; Millipore, Temecula, CA, USA).

Drug Application. During electrophysiological and fluorescence measurements, neurons were continuously superfused with normal extracellular solution, using a gravity-driven perfusion system, consisting of independent tubes for standard and agonist-containing (GABA, GCM) solutions, connected to a fast exchanger system (RSC-100; Bio-Logic) positioned 50-100 $\mu \mathrm{m}$ from the cell. Antagonists were usually acutely applied through a parallel tubes of the same perfusion system. DIOA, K252A and PP2 were pre-incubated for $1 \mathrm{~h}\left(37^{\circ} \mathrm{C}\right)$ and then continuously applied during the experiments. Sulfasalazine, APV/NBQX, BAPTA-AM and tetrodotoxin citrate were applied during co-culture in the co-culture medium and not superfused during recordings $(24 \mathrm{~h})$. The values of EGABA and RMP of control neurons, following different drug treatments are reported in Supplementary Table S2 and Supplementary Table S4.

Data analysis. Data, analyzed offline, are presented as mean \pm S.E.M.; we used the QuantityOne (Bio-Rad Laboratories S.r.l.) program for the densitometric analysis of all immunoblots. Origin 7 (Origin software; Microcal Software, Northampton, MA, USA) and Sigmaplot 11 (Systat Software Inc, London, UK) software were used for statistical analysis. Paired and unpaired $t$-test and one-way ANOVA were used for parametrical data, as indicated; Tukey test was used as post hoc test; Mann and Withney test for non parametrical data. We constructed I-V plots, cumulative distribution plots and fitted data points by linear or non-linear regression analysis using Origin software. Statistical significance for cumulative distributions was assessed with Kolmogorov-Smirnov test.

\section{Conflict of Interest}

The authors declare no conflict of interests.

Acknowledgements. This study is dedicated to the memory of Professor Fabrizio Eusebi, who initiated and inspired this direction of research. The work was supported by grants from PRIN 2009 (to DR, CL and SDA) and AIRC to CL. EM was supported by $\mathrm{PhD}$ programme in Neurophysiology, Sapienza University, Rome. Authors wish to thank Dr. Stefano Sensi for critical reading of the manuscript and precious suggestions.

\section{Author contributions}

SDA designed, carried out and analyzed all perforated patch clamp experiments; SC, FS and MGM performed whole-cell recordings; $\mathrm{CL}$ and $\mathrm{CL}$ designed, carried out and analyzed western blot experiments. Imaging experiments were designed, carried out and analyzed by EM, CB, PB and DR; DR and SDA conceived the project and wrote the manuscript with the help of PB and original input from Fabrizio Eusebi.

1. Moots PL, Maciunas RJ, Eisert DR, Parker RA, Laporte K, Abou-Khalil B. The course of seizure disorders in patients with malignant gliomas. Arch Neurol 1995; 52: 717-724.

2. Khan T, Akhtar W, Wotton CJ, Hart Y, Turner MR, Goldacre MJ. Epilepsy and the subsequent risk of cerebral tumour: record linkage retrospective cohort study. J Neurol Neurosurg Psychiatry 2011; 82: 1041-1045.

3. Sontheimer H. Malignant gliomas: perverting glutamate and ion homeostasis for selective advantage. Trends Neurosci 2003; 26: 543-549.

4. Cavus I, Kasoff WS, Cassaday MP, Jacob R, Gueorguieva R, Sherwin RS et al. Extracellular metabolites in the cortex and hippocampus of epileptic patients. Ann Neurol 2005; 57 : 226-235.

5. Köhling R, Senner V, Paulus W, Speckmann EJ. Epileptiform activity preferentially arises outside tumor invasion zone in glioma xenotransplants. Neurobiol Dis 2006; 22: 64-75.

6. Buckingham SC, Campbell SL, Haas BR, Montana V, Robel S, Ogunrinu T et al. Glutamate release by primary brain tumors induces epileptic activity. Nat Med 2011; 17: 1269-1274.

7. Lee HH, Deeb TZ, Walker JA, Davies PA, Moss SJ. NMDA receptor activity downregulates KCC2 resulting in depolarizing GABAA receptor-mediated currents. Nat Neurosci 2011; 14: 736-743.

8. Blaesse $\mathrm{P}$, Airaksinen MS, Rivera C, Kaila K. Cation-chloride cotransporters and neuronal function. Neuron 2009; 61: 820-838.

9. Cohen I, Navarro V, Clemenceau S, Baulac M, Miles R. On the origin of interictal activity in human temporal lobe epilepsy in vitro. Science 2002; 298: 1418-1421.

10. Huberfeld G, Wittner L, Clemenceau S, Baulac M, Kaila K, Miles R et al. Perturbed chloride homeostasis and GABAergic signaling in human temporal lobe epilepsy. J Neurosci 2007; 27: 9866-9873.

11. Palma E, Amici M, Sobrero F, Spinelli G, Di Angelantonio S, Ragozzino D et al. Anomalous levels of $\mathrm{Cl}$ - transporters in the hippocampal subiculum from temporal lobe epilepsy patients. make GABA excitatory. Proc Natl Acad Sci USA 2006; 103: 8465-8468.

12. Barmashenko G, Hefft S, Aertsen A, Kirschstein T, Köhling R. Positive shifts of the GABAA receptor reversal potential due to altered chloride homeostasis is widespread after status epilepticus. Epilepsia 2011; 52: 1570-1578.

13. Conti L, Palma E, Roseti C, Lauro C, Cipriani R, de Groot M et al. Anomalous levels of $\mathrm{Cl}$ - transporters cause a decrease of GABAergic inhibition in human peritumoral epileptic cortex. Epilepsia 2011; 52: 1635-1644.

14. Sciaccaluga M, Fioretti B, Catacuzzeno L, Pagani F, Bertollini C, Rosito M et al. CXCL12induced glioblastoma cell migration requie intermediate conductance $\mathrm{Ca2}$--activated K+ channel activity. Am J Physiol Cell Physiol 2010; 299: 175-184.

15. Markova O, Mukhtarov M, Real E, Jacob Y, Bregestovski P. Genetically encoded chloride indicator with improved sensitivity. J Neurosci Methods 2008; 170: 67-76.

16. Waseem T, Mukhtarov M, Buldakova S, Medina I, Bregestovski P. Genetically encoded $\mathrm{Cl}$-Sensor as a tool for monitoring of $\mathrm{Cl}$-dependent processes in small neuronal compartments. J Neurosci Methods 2010; 193: 14-23.

17. Bertollini C, Murana E, Mosca L, D'Erme M, Scala F, Francioso A et al. Transient increase in neuronal chloride concentration by neuroactive amino acids released from glioma cells. Front Mol Neurosci 2012; 5: 100

18. Hershfinkel M, Kandler K, Knoch ME, Dagan-Rabin M, Aras MA, Abramovitch-Dahan C et al. Intracellular zinc inhibits KCC2 transporter activity. Nat Neurosci 2009; 12: 725-727.

19. Chorin E, Vinograd O, Fleidervish I, Gilad D, Herrmann S, Sekler I et al. Upregulation of KCC2 activity by zinc-mediated neurotransmission via the mZnR/GPR39 receptor. J Neurosci 2011; 31: 12916-12926.

20. Rivera C, Voipio J, Thomas-Crusells J, Li H, Emri Z, Sipilä S et al. Mechanism of activity-dependent downregulation of the neuron-specific $\mathrm{K}-\mathrm{Cl}$ cotransporter $\mathrm{KCC}$. J Neurosci 2004; 24: 4683-4691.

21. Wake H, Watanabe M, Moorhouse AJ, Kanematsu T, Horibe S, Matsukawa N et al. Early changes in KCC2 phosphorylation in response to neuronal stress result in functional downregulation. J Neurosci 2007; 27: 1642-1650.

22. Huang YZ, Pan E, Xiong ZQ, McNamara JO. Zinc-mediated transactivation of TrkB potentiates the hippocampal mossy fiber-CA3 pyramid synapse. Neuron 2008; 57: 546-558.

23. Sensi SL, Paoletti P, Bush AI, Sekler I. Zinc in the physiology and pathology of the CNS. Nat Rev Neurosci 2009; 10: 780-791.

24. Frederickson CJ, Koh JY, Bush Al. The neurobiology of zinc in health and disease. Nat Rev Neurosci 2005; 6: 449-462.

25. Sensi SL, Ton-That $\mathrm{D}$, Weiss JH. Mitochondrial sequestration and $\mathrm{Ca}(2+)$-dependent release of cytosolic $\mathrm{Zn}(2+)$ loads in cortical neurons. Neurobiol Dis 2002; 10: 100-108. 
26. Wardle RA, Poo MM. Brain-derived neurotrophic factor modulation of GABAergic synapses by postsynaptic regulation of chloride transport. J Neurosci 2003; 23: 8722-8732.

27. Medina I, Friedel P, Rivera C, Kahle KT, Kourdougli N, Uvarov P et al. Current view on the functional regulation of the neuronal $\mathrm{K}(+)-\mathrm{Cl}(-)$ cotransporter KCC2. Front Cell Neurosci 2014; 8: 27.

28. Kahle KT, Deeb TZ, Puskarjov M, Silayeva L, Liang B, Kaila K et al. Modulation of neuronal activity by phosphorylation of the K-Cl cotransporter KCC2. Trends Neurosci 2013; 36: 726-737.

29. Ferrini F, De Koninck Y. Microglia control neuronal network excitability via BDNF signalling. Neural Plast 2013; 2013: 429815.

30. Dineley KE, Devinney MJ 2nd, Zeak JA, Rintoul GL, Reynolds IJ. Glutamate mobilizes [Zn2t] through Ca2+-dependent reactive oxygen species accumulation. J Neurochem 2008; 106: 2184-2193.

31. McCord MC, Aizenman E. The role of intracellular zinc release in aging, oxidative stress, and Alzheimer's disease. Front Aging Neurosci 2014; 6: 77.

32. Sensi SL, Paoletti P, Koh JY, Aizenman E, Bush Al, Hershfinkel M. The neurophysiology and pathology of brain zinc. J Neurosci 2011; 31: 16076-16085.

33. Zatta P, Drago D, Bolognin S, Sensi SL. Alzheimer's disease, metal ions and metal homeostatic therapy. Trends Pharmacol Sci 2009; 30: 346-355.

34. Rosito M, Deflorio C, Limatola C, Trettel F. CXCL16 orchestrates adenosine A3 receptor and MCP-1/CCL2 activity to protect neurons from excitotoxic cell death in the CNS. J Neurosci 2012; 32: 3154-3163.

35. Campbell SL, Buckingham SC, Sontheimer H. Human glioma cells induce hyperexcitability in cortical networks. Epilepsia 2012; 53: 1360-1370.

36. Marcus HJ, Carpenter KL, Price SJ, Hutchinson PJ. In vivo assessment of high-grade glioma biochemistry using microdialysis: a study of energy-related molecules, growth factors and cytokines. J Neurooncol 2010; 1: 11-23.

37. Kettenmann H, Hanisch UK, Noda M, Verkhratsky A. Physiology of microglia. Physiol Rev 2011; 91: 461-553.

38. Buckingham SC, Robel S. Glutamate and tumor-associated epilepsy: glial cell dysfunction in the peritumoral environment. Neurochem Int 2013; 63: 696-701.
39. Lauro C, Cipriani R, Catalano M, Trettel F, Chece G, Brusadin V et al. Adenosine A1 receptors and microglial cells mediate $\mathrm{CX} 3 \mathrm{CL} 1$-induced protection of hippocampal neurons against Glu-induced death. Neuropsychopharmacology 2010; 35: 1550-1559.

40. D'Onofrio M, Arcella A, Bruno V, Ngomba RT, Battaglia G, Lombari V et al. Pharmacological blockade of mGlu2/3 metabotropic glutamate receptors reduces cell proliferation in cultured human glioma cells. J Neurochem 2003; 84: 1288-1295.

41. D'Alessandro G, Catalano M, Sciaccaluga M, Chece G, Cipriani R, Rosito M et al. KCa3.1 channels are involved in the infiltrative behavior of glioblastoma in vivo. Cell Death Dis 2013; 4: e773.

42. Cantini G, Pisati F, Pessina S, Finocchiaro G, Pellegatta S. Immunotherapy against the radial glia marker GLAST effectively triggers specific antitumor effectors without autoimmunity. Oncoimmunology 2012; 1: 884-893.

43. Akaike N. Gramicidin perforated patch recording and intracellular chloride activity in excitable cells. Prog Biophys Mol Biol 1996; 65: 251-264.

44. Autere AM, Lamsa K, Kaila K, Taira T. Synaptic activation of GABAA receptors induces neuronal uptake of Ca2+ in adult rat hippocampal slices. J Neurophysiol 1999; 81: $811-816$.

45. Lauro C, Di Angelantonio S, Cipriani R, Sobrero F, Antonilli L, Brusadin V et al. Activity of adenosine receptors type 1 is required for CX3CL1-mediated neuroprotection and neuromodulation in hippocampal neurons. J Immunol 2008; 180: 7590-7596.

(1) Cell Death and Disease is an open-access journal published by Nature Publishing Group. This work is licensed under a Creative Commons Attribution 3.0 Unported License. The images or other third party material in this article are included in the article's Creative Commons license, unless indicated otherwise in the credit line; if the material is not included under the Creative Commons license, users will need to obtain permission from the license holder to reproduce the material. To view a copy of this license, visit http:// creativecommons.org/licenses/by/3.0/

Supplementary Information accompanies this paper on Cell Death and Disease website (http://www.nature.com/cddis) 